\title{
A PROOF OF THE JORDAN CURVE THEOREM
}

\section{HELGE TVERBERG}

\section{Introduction}

Let $\Gamma$ be a Jordan curve in the plane, i.e. the image of the unit circle $C=\left\{(x, y) ; x^{2}+y^{2}=1\right\}$ under an injective continuous mapping $\gamma$ into $R^{2}$. The Jordan curve theorem [1] says that $R^{2} \backslash \Gamma$ is disconnected and consists of two components. (We shall use the original definition whereby two points are in the same component if and only if they can be joined by a continuous path (image of $[0,1])$ ).)

Although the JCT is one of the best known topological theorems, there are many, even among professional mathematicians, who have never read a proof of it. The present paper is intended to provide a reasonably short and selfcontained proof or at least, failing that, to point at the need for one.

\section{Prerequisites and lemmata}

Some elementary concepts and facts from analysis are needed, for instance uniform continuity. One must know that $\Gamma$ is compact, and so is any continuous path. Also, if $A$ and $B$ are disjoint compact sets, $\inf \{|a-b| ; a \in A, b \in B\}$, to be denoted by $d(A, B)$, is $>0$. Sometimes it is useful to keep in mind that $\gamma^{-1}$ is continuous. It would have been possible to avoid the use of these results, at the cost of an extra page, by replacing their applications by arguments ad hoc. The "deepèst" result needed would then be Weierstrass' theorem to the effect that any bounded sequence of real numbers has a convergent subsequence.

The main idea of the proof is to approximate $\Gamma$ by polygons, prove the theorem for these and then pass to the limit. This is a classical approach, and Lemmata 1 and 2 are of course well known. Lemmata 3 and 4 seem new, and of some independent interest. Their function is to quantify certain aspects of the polygonal case, so as to make the limit process work. The non-Jordan closed curves $\infty$ (upper half followed by lower half) and - (run through once in each direction) are both limits of Jordan polygons. The purpose of Lemmata 3 and 4 is to ensure that the bad things happening in these two cases can not happen to a Jordan curve.

A Jordan curve is said to be a Jordan polygon if $C$ can be covered by finitely many arcs on each of which $\gamma$ has the form: $\gamma(\cos t, \sin t)=(\lambda t+\mu, \rho t+\sigma)$ with constants $\lambda, \mu, \rho, \sigma$. Thus $\Gamma$ is a closed polygon without self intersections.

Lemma 1. The Jordan curve theorem holds for every Jordan polygon $\Gamma$.

Proof. Let $\Gamma$ have edges $E_{1}, \ldots, E_{n}$ and vertices $v_{1}, \ldots, v_{n}$ with

$$
E_{l} \cap E_{l+1}=\left\{v_{l}\right\}, i=1, \ldots, n, \quad\left(E_{n+1}=E_{1}, v_{n+1}=v_{n}\right) .
$$

We first prove that $E^{2} \backslash \Gamma$ has at most two components. Consider the sets $N_{i}=\left\{q ; d\left(q, E_{i}\right)<\delta\right\}$ where $\delta=\min \left\{d\left(E_{i}, E_{j}\right) ; 1<j-i<n-1\right\}$. It is then clear 
that $N_{i} \cap \Gamma \subset E_{i-1} \cup E_{i} \cup E_{i+1} \quad\left(E_{0}=E_{n}\right)$, and that $N_{i} \backslash \Gamma$ consists of two components, $N_{i}^{\prime}$ and $N_{i}^{\prime \prime}$, where we may assume

$$
N_{i}^{\prime} \cap N_{i+1}^{\prime} \neq \varnothing, \quad N_{i}^{\prime \prime} \cap N_{i+1}^{\prime \prime} \neq \varnothing, \quad i=1, \ldots, n .
$$

Then $N_{1}^{\prime} \cup \ldots \cup N_{n}^{\prime}$ and $N_{1}^{\prime \prime} \cup \ldots \cup N_{n}^{\prime \prime}$ are both connected sets and for any $p$ in $R^{2} \backslash \Gamma$ there is a line segment in $R^{2} \backslash \Gamma$ connecting $p$ to one of them.

In order to see that there are at least two components, we partition $R^{2} \backslash \Gamma$ into odd and even points and prove that no continuous path connects an odd point to an even one.

The partition is done as follows. Assume the coordinate system chosen such that when $v_{i}=\left(x_{i}, y_{i}\right)$ the $x_{i}$ are all different. For every $p=(x(p), y(p))$ in $R^{2} \backslash \Gamma$, let $m(p)$ be the number of points in which the upward vertical ray from $p$ meets $\Gamma$. We make the provision however, that if one (and hence only one) of these points is a vertex, $v_{i}$, say, then it is not counted if $v_{i-1}$ and $v_{i+1}$ lie to the same side of the vertical through $p$. If $m(p)$ is odd (even) we say that $p$ is odd (even).

Note that for every $p$ there is an $\varepsilon>0$ such that $q$ has the same parity as $p$ whenever $d(p, q)<\varepsilon$.

This is obvious if $x(p) \neq x_{i}$ for all $i$, and one even gets $m(q)=m(p)$. If $x(p)=x_{1}$, say, but $v_{1}$ lies below $p$, or $\left(x_{n}-x_{1}\right)\left(x_{2}-x_{1}\right)<0$, one also gets $m(q)=m(p)$. If, however, $v_{1}$ lies above $p$, with $v_{2}$ and $v_{n}$ both to the left, say, of $v_{1}$ one has $m(q)=m(p)$ only in the right half-disc where $x(q) \geqslant x_{1}$ while $m(q)=m(p)+2$ in the remainder of the disc $d(p, q)<\varepsilon$.

Finally it must be verified that if $\Pi$ is a continuous path, given by $\pi:[0,1] \rightarrow R^{2} \backslash \Gamma$, with $\pi(0)$ odd, say, then $\pi(1)$ is odd too. Put $t_{0}=\operatorname{lub}\{t ; \pi(t)$ is odd $\}$. As all points sufficiently near to an odd (even) point are odd (even), continuity shows that $t_{0}>0$, that $\pi\left(t_{0}\right)$ is odd, and that a contradiction would arise if $t_{0}<1$.

Lemma 2. Every Jordan curve $\Gamma$ can be approximated arbitrarily well by a Jordan polygon $\Gamma^{\prime}$.

Proof. We want $\left|\gamma-\gamma^{\prime}\right|<\varepsilon$, say. Choose $\varepsilon_{1}>0$ such that

$$
|p-q| \leqslant \varepsilon_{1} \quad \Rightarrow \quad|\gamma(p)-\gamma(q)|<\varepsilon / 2
$$

and then $\varepsilon_{2}>0$ such that

$$
|\gamma(p)-\gamma(q)| \leqslant \varepsilon_{2} \quad \Rightarrow \quad|p-q|<\min \left(\varepsilon_{1}, \sqrt{3}\right) .
$$

Put $\delta=\min \left(\varepsilon / 2, \varepsilon_{2}\right)$.

Now $\Gamma$ meets only finitely many of the squares

$$
\{(x, y) ;|x-k \delta / \sqrt{2}| \leqslant \delta / 2 \sqrt{2},|y-| \delta / \sqrt{2} \mid \leqslant \delta / 2 \sqrt{2}\}, \quad(k, l \text { integers }) .
$$

Let these be $S_{1}, \ldots, S_{n}$. Because $\delta \leqslant \varepsilon_{2}$, each set $\gamma^{-1}\left(S_{i}\right)$ has diameter less than $\sqrt{3}$ and it is thus contained in a unique minimal circle arc $A_{i}$, shorter than $2 \pi / 3$.

We first change $\Gamma_{0}=\Gamma$ into another Jordan curve $\Gamma_{1}$ by putting $\gamma_{1}=\gamma_{0}\left(=\gamma^{\prime}\right)$ outside $A_{1}, \gamma_{1}(\cos t, \sin t)=(\lambda t+\mu, \rho t+\sigma)$ when $(\cos t, \sin t)$ is a point on $A_{1}$. Here 
$\lambda, \mu, \rho, \sigma$ are chosen so that $\gamma_{1}$ becomes continuous. Note that when $i \geqslant 2, \gamma_{1}^{-1}\left(S_{i}\right)$ is contained in $\gamma_{0}^{-1}\left(S_{i}\right)$, and thus has diameter less than $\sqrt{3}$; it can even be empty.

The next step is to straighten $\gamma_{1}\left(A_{2}\right)$, where $A_{2}$ is the minimal arc containing $\gamma_{1}^{-1}\left(S_{2}\right)$, in the same way to obtain $\Gamma_{2}$. If $\gamma_{1}^{-1}\left(S_{2}\right)=\varnothing$, put $\Gamma_{2}=\Gamma_{1}$. Continuing, we arrive at the Jordan polygon $\Gamma_{n}$.

Consider now any $a$ for which $\gamma_{n}(a) \neq \gamma(a)$. There is an $i$ so that $\gamma_{n}(a)=\gamma_{i}(a) \neq \gamma_{i-1}(a)$. By construction $a$ belongs to the $\operatorname{arc} A_{i}$, with endpoints $b, c$, say. Note that the construction of the $\Gamma_{j}$ gives $\gamma_{i}(b)=\gamma(b), \gamma_{i}(c)=\gamma(c)$. Then

$$
\begin{aligned}
\left|\gamma(a)-\gamma_{n}(a)\right| & =\left|\gamma(a)-\gamma(b)+\gamma_{i}(b)-\gamma_{i}(a)\right| \\
& \leqslant|\gamma(a)-\gamma(b)|+\delta \\
& \leqslant|\gamma(a)-\gamma(b)|+\varepsilon / 2 .
\end{aligned}
$$

As $|a-b|<|c-b| \leqslant \varepsilon_{1}$, because $|\gamma(b)-\gamma(c)| \leqslant \delta \leqslant \varepsilon_{2}$, we have $|\gamma(a)-\gamma(b)|<\varepsilon / 2$, showing that $\left|\gamma(a)-\gamma_{n}(a)\right|<\varepsilon$.

Lemma 3. Let $\Gamma$ be a Jordan polygon. Then the bounded component of $R^{2} \backslash \Gamma$ contains a disc, on the boundary circle of which are two points $\gamma(a)$ and $\gamma(b)$, with $|a-b| \geqslant \sqrt{3}$.

Proof. There clearly exists a disc $D$ as described with $|a-b|$ maximal. Assume $|a-b|<\sqrt{3}$. Then $a$ and $b$ are the endpoints of an $\operatorname{arc} A$, of length $>(4 / 3) \pi$. The boundary circle of $D$ can not meet $\gamma(A) \backslash\{\gamma(a), \gamma(b)\}$ as $\max \{|a-c|,|b-c|\}>|a-b|$ for every $c$ in $A \backslash\{a, b\}$. Let $\gamma\left(v_{1}\right), \ldots, \gamma\left(v_{n}\right)$ be the vertices of $\Gamma$ in $\gamma(A)$, as met when passing from $\gamma(a)$ to $\gamma(b)$. If $v_{1} \neq a$ and $v_{n} \neq b$, then a circle touching the segments $\gamma(a) \gamma\left(v_{1}\right)$ and $\gamma(b) \gamma\left(v_{n}\right)$ very near $\gamma(a)$ and $\gamma(b)$, in points $\gamma\left(a^{\prime}\right)$ and $\gamma\left(b^{\prime}\right)$, will meet $\Gamma$ in these points only. As $\left|a^{\prime}-b^{\prime}\right|>|a-b|$ a contradiction is obtained. If, say, $v_{1} \neq a, v_{n}=b$ a circle touching $\gamma(a) \gamma\left(v_{1}\right)$ very near $\gamma(a)$ and passing through $b$ will do. If $v_{1}=a, v_{n}=b$ we consider a variable circle through $\gamma(a)$ and $\gamma(b)$ as its centre moves from the centre of $D$ into the domain bounded by the radii to $\gamma(a)$ and $\gamma(b)$ together with $\gamma(A)$, (use Lemma 1). Eventually the circle either meets $\gamma(A)$ in points other than $\gamma(a), \gamma(b)$ or becomes tangent to one of the segments $\gamma(a) \gamma\left(v_{2}\right), \gamma(b) \gamma\left(v_{n-1}\right)$. Contradiction arises again and the proof is finished.

Consider now a Jordan polygon $\Gamma$ and two points $a, b$, belonging to the same component, $X$, of $R^{2} \backslash \Gamma$. For every chord $S$, contained in $X$ except for its endpoints, $X \backslash S$ consists of two components, as is immediate from Lemma 1. Let the distance between $\Gamma$ and $\{a, b\}$ be at least 1 and assume that for every $S$ of length less than 2 , $a$ and $b$ are in the same component of $X \backslash S$. Then we have

Lemma 4. Under the assumptions stated there is a continuous path $\Pi$ from a to $b$ such that $d(\Pi, \Gamma) \geqslant 1$.

Proof. We first note that if $a^{\prime}$ is any point in $R^{2}$, connected to $a$ by a continuous path $\Pi^{\prime}$, where $d\left(\Pi^{\prime}, \Gamma\right) \geqslant 1$, then $a^{\prime}$ and $b$ satisfy the conditions put on $a$ and $b$. For let $S$ be a chord to $\Gamma$, of length less than 2 . Then $a$ and $a^{\prime}$ are in the same component of $X \backslash S$, as $S$ does not meet $\Pi^{\prime}$, and so are $a$ and $b$, by assumption. 
By the preceding paragraph we may now assume that $d(a, \Gamma)=d(b, \Gamma)=1$. Choose $u_{a}$ and $u_{b}$ on $C$ such that $\left|\gamma\left(u_{a}\right)-a\right|=\left|\gamma\left(u_{b}\right)-b\right|=1$. Let $D$ be a mobile unit circle, initially placed with $c$, its centre, in $a$. The desired path $\Pi$ will be obtained as the curve followed by $c$ as $D$ (confined to $X \cup \Gamma$ ) rolls along $\Gamma$ in the obvious way, starting at $\gamma\left(u_{a}\right)$, until $c$ falls in $b$.

As $D$, in most cases, skips certain parts of $\Gamma$ we first check that $D$ at least arrives at $\gamma\left(u_{b}\right)$. If it doesn't then, for some position of $D, \Gamma$ and $D$ have a common chord $S=\gamma\left(u_{1}\right) \gamma\left(u_{2}\right)$ of length less than 2 , so that $X \backslash S$ consists of two components $Y$ and $Z$. Furthermore $c$ is in $Y$, say, while $\gamma\left(u_{b}\right)$ is on the boundary of $Z$, somewhere on $\Gamma$, strictly between $\gamma\left(u_{1}\right)$ and $\gamma\left(u_{2}\right)$.

By the first paragraph above $b$ is in $Y$. Consider $E$, the unit circle centred at $b$, and its radius towards $\gamma\left(u_{b}\right)$. This radius starts in $Y$ and later runs in $Z$ until it meets $\Gamma$ in $\gamma\left(u_{b}\right)$. Hence it crosses $S$, and we find also that $b$ and $c$ lie to the same side of the line through $S$.

Now, as $E$ encompasses neither $\gamma\left(u_{1}\right)$ nor $\gamma\left(u_{2}\right), E$ must intersect $S$ in two points. With $b$ and $c$ being to the same side of $S$, the radius to $\gamma\left(u_{b}\right)$ must stop inside or on $D$ and so does not end in $\gamma\left(u_{b}\right)$ as it should.

Having seen that $D$ reaches $\gamma\left(u_{b}\right)$, we must verify that $c$ reaches $b$, too. If $\gamma\left(u_{b}\right)$ is not a vertex of $\Gamma$, there is no problem. A problem arises only as follows: $D$ and $\Gamma$ have a common chord $S$ of length less than 2 , with $\gamma\left(u_{b}\right)$ as one endpoint, and pointing into the angle $c \gamma\left(u_{b}\right) b$. But then the segment $b c$ meets $S$ in one point and avoids $\Gamma$, so that $b$ and $c$ are in different components of $X \backslash S$. Now use the first paragraph of our proof again, to end the last one.

\section{Proof of the theorem}

$R^{2} \backslash \Gamma$ has at least two components:

There is one unbounded component. The existence of a bounded one is proved as follows. Draw a large circle $C_{0}$ around $\Gamma$, and let $\Gamma_{1}, \Gamma_{2}, \ldots$, be a sequence of Jordan polygons converging to $\Gamma$. For each $\Gamma_{n}$ there is a circle $C_{n}$ as given by Lemma 3 , containing points $\gamma_{n}\left(a_{n}\right)$ and $\gamma_{n}\left(b_{n}\right)$, with $\left|a_{n}-b_{n}\right| \geqslant \sqrt{3}$, and having centre $z_{n}$. Passing to a subsequence of the original one we may assume that all the $\Gamma_{n}$ are inside $C_{0}$ and that $z_{n} \rightarrow z$, say, as $n \rightarrow \infty$.

Now choose $\varepsilon>0$ such that $|a-b| \geqslant \sqrt{3} \Rightarrow|\gamma(a)-\gamma(b)| \geqslant \varepsilon$. Then $\left|\gamma\left(a_{n}\right)-\gamma\left(b_{n}\right)\right| \geqslant \varepsilon$ so that $\left|\gamma_{n}\left(a_{n}\right)-\gamma_{n}\left(b_{n}\right)\right|>\varepsilon / 2$ for large $n$. Thus diameter $\left(C_{n}\right)>\varepsilon / 2$, so that $d\left(z_{n}, \Gamma_{n}\right)>\varepsilon / 4$. This shows that for large $n, z$ and $z_{n}$ are in the same component of $R^{2} \backslash \Gamma_{n}$ (and of $R^{2} \backslash \Gamma$ ).

If now $z$ is in the unbounded component of $R^{2} \backslash \Gamma$ there is a continuous path $\Pi$, in $R^{2} \backslash \Gamma$, connecting $z$ to a point outside $C_{0}$. Put $d(\Pi, \Gamma)=\delta$. For large $n,\left|\gamma_{n}-\gamma\right|<\delta / 2$ and then $d\left(\Pi, \Gamma_{n}\right)>\delta / 2$ so that, for very large $n, z$ and $z_{n}$ are in the unbounded component of $R^{2} \backslash \Gamma_{n}$. This contradicts the definition of $z_{n}$.

$R^{2} \backslash \Gamma$ has at most two components:

Assume $p, q$ and $r$ to be points from three distinct components of $R^{2} \backslash \Gamma$, with $d(\Gamma,\{p, q, r\})=\varepsilon$. Let $\Gamma_{1}, \Gamma_{2}, \ldots$ converge to $\Gamma$, as before. Then $d\left(\Gamma_{n},\{p, q, r\}\right) \geqslant \varepsilon / 2$ for large $n$ and two of the three points have to be in the same component $X_{n}$ of $R^{2} \backslash \Gamma_{n}$. Passing to a subsequence, we may assume that $p$ and $q$ are in $X_{n}$ for all $n$.

Assume first that there is a $\delta \in(0, \varepsilon)$ and infinitely.many $n$ such that $p$ is connected to $q$ by a continuous path $\Pi_{n}$, with $d\left(\Gamma_{n}, \Pi_{n}\right) \geqslant \delta$. For such large $n, d\left(\Gamma, \Pi_{n}\right)>\delta / 2$, so that $p$ and $q$ would be in the same component of $R^{2} \backslash \Gamma$. Hence there is no such $\delta$. 
We now apply Lemma 4. The non-existence of $\delta$ yields a sequence of chords $S_{1}, S_{2}, \ldots$, and an increasing sequence $n(1), n(2), \ldots$ so that one has

(i) $p$ and $q$ are in different components of $X_{n(i)} \backslash S_{i}$.

(ii) As $i \rightarrow \infty, \gamma_{n(i)}\left(a_{i}\right)-\gamma_{n(i)}\left(b_{i}\right) \rightarrow 0$, where $\gamma_{n(i)}\left(a_{i}\right)$ and $\gamma_{n(i)}\left(b_{i}\right)$ are the endpoints of $S_{i}$.

Now, as $i \rightarrow \infty, \gamma\left(a_{i}\right)-\gamma\left(b_{i}\right) \rightarrow 0$, so that $a_{i}-b_{i} \rightarrow 0$. For infinitely many $i, p$, say, belongs to the component of $X_{n(i)} \backslash S_{i}$ bounded by $S_{i}$ and $\gamma_{n(i)}\left(A_{i}\right)$ where $A_{i}$ is the small arc on $C$ with endpoints $a_{i}$ and $b_{i}$. As $a_{i}-b_{i} \rightarrow 0$, diameter $\gamma_{n(i)}\left(A_{i}\right) \rightarrow 0$, so that the diameter of the component just defined is smaller than $\varepsilon$ for large $i$. In particular we have $\left|p-\gamma\left(a_{i}\right)\right|<\varepsilon$, the contradiction needed to finish the proof.

As more or less free byproducts of the above proof we obtain two theorems. The first is well known, but the second one is possibly new.

THEOREM 1. Let $\Gamma$ be a Jordan arc, i.e. the image of $[0,1]$ under an injective continuous mapping into $R^{2}$. Then $R^{2} \backslash \Gamma$ is connected.

Proof. Modify Lemmata 1,2 and 4 and the second part of the above proof. The first part gives

Theorem 2. Let $\Gamma$ be a Jordan curve. Put $\delta=\min \{|\gamma(p)-\gamma(q)| ;|p-q| \geqslant \sqrt{3}\}$. Then the bounded component of $R^{2} \backslash \Gamma$ contains an open disc of diameter $\delta$.

The reader might like to formulate his own version of a generalized Lemma 4. There are also higher-dimensional analogues to speculate about.

\section{Reference}

1. C. Jordan, Cours d'Analyse de lÉcole Polytechnique (Gauthier-Villars, Paris, 1887), vol. 3, 587-594.

University of Bergen,

Bergen, Norway. 